\title{
The Cumulative Incidence and Risk Factors of Recurrent Venous Thromboembolism in the Elderly
}

This article was published in the following Dove Press journal:

Vascular Health and Risk Management

\author{
Fahad AS Aleidan (ID) ${ }^{1,2}$ \\ 'College of Medicine, King Saud Bin \\ Abdulaziz University for Health Sciences, \\ Riyadh, Saudi Arabia; ${ }^{2}$ Anticoagulation \\ Clinic, King Abdulaziz Medical City, \\ Riyadh, Saudi Arabia
}

Background: Incidence and outcomes of recurrent venous thromboembolism (VTE) in the elderly are still not fully elucidated. The purpose of this study was to determine the incidence and identify the risk factors of VTE recurrence in this population.

Methods: A prospective cohort study of a one-year follow-up of 277 patients aged $\geq 65$ years with primary VTE was performed at King Abdulaziz Medical City, a tertiary care teaching hospital in Riyadh, Saudi Arabia. Demographic data, risk factors, and the consequences of VTE (recurrence, bleeding, and mortality) were recorded.

Results: Of the 277 VTE patients, 39 (14\%) were diagnosed with recurrent VTE over a median follow-up period of 12 months. The cumulative incidence of recurrent VTE was 12.75 per hundred patient-year (95\% CI, 8.24-17.36). In multivariate Cox regression, malignancy (hazard ratio [HR], 2.87, 95\% CI, 1.32-6.24, $p=0.008$ ) and surgery (HR 2.78, $95 \%$ CI, 1.36-5.67, $p=0.005)$ were identified as independent risk factors for recurrent VTE. Metformin had a significant independent protection effect (HR, 0.16, 95\% CI, 0.08-0.33, $p<0.001)$. During follow-up, two patients in the recurrent VTE group and five patients in the group with no recurrent VTE, all of whom were minor bleeding cases, reported no major bleeding. Seven $(18 \%)$ patients in the recurrent VTE group and nine $(4 \%)$ patients in the group with no recurrent VTE died $(\mathrm{p}<0.001)$.

Conclusion: The findings of this study show that elderly patients with initial VTE have a recurrent rate $(14 \%)$, with a cumulative incidence rate of 12.75 per hundred patient-year. Malignancy and surgery were the most important clinical risk factors to impact significantly the development of recurrent VTE in our elderly population. Metformin may have a protective effect against recurrent VTE in the elderly population, and a larger study is needed to validate our findings.

Keywords: elderly, incidence, recurrence, risk factors, venous thromboembolism

\section{Introduction}

Venous thromboembolism (VTE) constitutes a spectrum of events ranging from asymptomatic distal deep venous thrombosis (DVT) and subsegmental pulmonary embolism (PE), to limb threatening DVT and fatal PE. ${ }^{1}$ The annual incidence of VTE ranges from 1.5 to 3.0 cases per 1,000 individuals, and is considered the third most common cause of cardiovascular disease and death after myocardial infarction and stroke. ${ }^{1,2}$ The risk of VTE and its recurrence is higher in the population aged 65 years or more, and the risk in patients above 70 years old are four to six times higher than in younger patients; additionally, this risk doubles with each decade of ageing. ${ }^{2,3}$ Importantly, elderly patients also exhibit a higher mortality rate due to

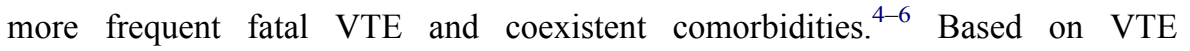

Correspondence: Fahad AS Aleidan College of Medicine, King Saud Bin Abdulaziz University for Health Sciences, Riyadh, Saudi Arabia

Tel +966II80IIIII

Email faleidan@gmail.com 
stratification, the risk of recurrent provoked VTE by temporary risk factors such as surgery or pregnancy is lower than when VTE was unprovoked. ${ }^{7-9}$ The risk of recurrence among VTE patients is influenced also by sex, as males have a significantly higher risk of developing a second episode of VTE than females. ${ }^{10-12}$ Although recurrent VTE is an important cause of morbidity and mortality among the elderly, little has been written about the risk factors predisposing to recurrent VTE in the elderly. The aims of our study were to assess the possible risk factors associated with recurrent VTE in a prospective cohort of elderly patients, and to determine the cumulative incidence of recurrence rate and the frequency of bleeding and mortality.

\section{Patients and Methods}

In this single-centre, prospective cohort analysis, elderly patients aged 65 years and above with first episode of objectively confirmed VTE, deep vein thrombosis, or pulmonary embolism were identified between 1 February 2016 and 31 December 2019, in the inpatient and outpatient settings at King Abdulaziz Medical City, Health Affairs, Ministry of National Guard, Riyadh, Saudi Arabia. Objectively confirmed VTE is defined as: a symptomatic VTE confirmed by ultrasound imaging for DVT and spiral computed tomography (CT) imaging for PE. Surgical intervention, thrombolytic therapy used, terminal illnesses, or severe dementia were an exclusion criterion. The following demographic characteristics including age, sex, weight, and height were recorded. Site of index VTE, category of index VTE, initial anticoagulant agents, duration of anticoagulation, medication currently used (proton pump inhibitors, antiplatelet, metformin, and statins therapy), comorbidities (hypertension, diabetes mellitus, COPD, dyslipidaemia, inflammatory bowel disease, chronic heart failure, and chronic kidney diseases), imaging reports (CT scan or Doppler ultrasound), and laboratory findings (Haemoglobin and international normalised ratios) were abstracted using standardised data collection spread sheets. All events were objectively reviewed during the inpatient stay or outpatient visit face-to-face, and periodic reviews were made of patients' electronic medical records.

A prospective follow-up was continued for 12 months to assess the main outcome variables over the study period. Recurrent VTE (DVT or PE) was considered when confirmed by the same imaging methods and documented in the electronic medical record, and the treating clinician had resumed anticoagulant therapy for at least a period of three months. Death and bleeding occurrence are assessed and reported; major bleeding in non-surgical patients is defined as fatal bleeding, and/or symptomatic bleeding in a critical area or organ, such as intracranial, intraspinal, intraocular, retroperitoneal, intraarticular or pericardial, or intramuscular with compartment syndrome, and/or requiring a transfusion of at least two units of packed red blood cells, and/or reduction in haemoglobin level of more than $20 \mathrm{gm} / \mathrm{L}^{13}$

\section{Statistical Analysis}

Baseline characteristics were compared using student$t$-test for continuous variables and chi-square test for categorical variables as appropriate. The Kaplan-Meier method was used to construct VTE recurrence probabilities. All recruited patients were available for analysis, and were censored at time of recurrent event, death, loss to follow-up, or end of one-year follow-up. Univariate and multivariate Cox proportional hazard regression models were fitted to determine factors associated with hazard of VTE recurrence. These factors included: immobilisation, malignancy, obesity, surgery, and use of metformin. All tests were two-sided and a $p$-value $<0.05$ was considered significant.

\section{Result}

Of the 324 initial recruited patients with initial VTE, 47 patients were excluded because they had one or more exclusion criterion. The final sample size consisted of 277 patients. Overall, the mean $( \pm \mathrm{SD})$ age was $70( \pm 4)$ years and $52 \%$ were males. Based on the site of index VTE, 168 (61\%) patients had PE (with or without DVT), $90(32 \%)$ had proximal DVT, and 19 (7\%) had isolated distal DVT. The majority of patients in both groups were treated with warfarin $(77 \%$ vs $75 \%)$. The demographic and clinical characteristics of the study population are shown in Table 1.

Figure 1 shows the Kaplan-Meier recurrence probabilities curve plot. Overall, VTE recurrence rate was $14 \%$ (39/238) with cumulative incidence rate of 12.75 per hundred patient-year (95\% CI, 8.24-17.36). The cumulative incidence was further analysed according to the site of index VTE and the categorisation of index VTE. The cumulative incidences of recurrent VTE per hundred patient-year was 7.51 (95\% CI, 2.35-12.68) for patients who developed PE with or without DVT, 4.37 (95\% CI, 0.29-8.20) for patients with proximal DVT, and $0.98(95 \%$ 
Table I Baseline Characteristics of the Patients

\begin{tabular}{|c|c|c|c|}
\hline Variables & $\begin{array}{l}\text { Recurrent } \\
\text { VTE } \\
(n=39)\end{array}$ & $\begin{array}{l}\text { No } \\
\text { Recurrent } \\
\text { VTE } \\
(n=238)\end{array}$ & $P$ value \\
\hline \multicolumn{4}{|l|}{ Age, year } \\
\hline Mean $( \pm S D)$ & $69( \pm 7.7)$ & $70( \pm 8.2)$ & 0.730 \\
\hline Male sex, no. (\%) & $20(5 I)$ & $124(52)$ & 0.567 \\
\hline \multicolumn{4}{|l|}{$\mathrm{BMI}, \mathrm{kg} / \mathrm{m}^{2}$} \\
\hline Mean $( \pm S D)$ & $29( \pm 7.3)$ & $27( \pm 8.2)$ & 0.535 \\
\hline \multicolumn{4}{|l|}{ Co-morbidity, no. (\%) } \\
\hline DM & $30(78)$ & $198(83)$ & 0.575 \\
\hline HTN & 31 (79) & $196(82)$ & 0.309 \\
\hline COPD & $10(26)$ & $66(24)$ & 0.825 \\
\hline Dyslipidemia & $22(56)$ & $140(59)$ & 0.200 \\
\hline Inflammatory bowel disease & $5(13)$ & $28(12)$ & 0.747 \\
\hline Chronic heart failure & $8(20)$ & $52(22)$ & 0.944 \\
\hline Chronic Kidney diseases & $4(10)$ & $25(\mathrm{II})$ & 0.966 \\
\hline \multicolumn{4}{|l|}{ Site of index VTE, no. (\%) } \\
\hline Isolated distal DVT & $3(8)$ & $16(7)$ & 0.836 \\
\hline Proximal DVT & $13(33)$ & $77(32)$ & 0.931 \\
\hline PE with/without DVT & $23(59)$ & $145(61)$ & 0.908 \\
\hline \multicolumn{4}{|c|}{$\begin{array}{l}\text { Categorization of index VTE, no. } \\
\text { (\%) }\end{array}$} \\
\hline \multicolumn{4}{|l|}{ Provoked } \\
\hline Malignancy-related VTE & II (28) & $18(8)$ & $<0.001$ \\
\hline Immobilization (>3 days) & $25(64)$ & III (47) & 0.043 \\
\hline Obesity, BMI $\geq 30 \mathrm{~kg} / \mathrm{m}^{2}$ & $12(31)$ & $41(17)$ & 0.046 \\
\hline Surgery & $23(59)$ & $63(26)$ & $<0.001$ \\
\hline Unprovoked & $6(15)$ & $19(8)$ & 0.135 \\
\hline \multicolumn{4}{|l|}{ Outcomes } \\
\hline Bleeding* & $2(5 \%)$ & $5(2 \%)$ & 0.281 \\
\hline Death & $7(18 \%)$ & $9(4 \%)$ & $<0.001$ \\
\hline
\end{tabular}

Note: *All were minor bleeding.

Abbreviations: BMI, body mass index; COPD, chronic obstructive pulmonary disease; DM, diabetes mellitus, DVT, deep vein thrombosis; PE, pulmonary embolism; VTE, venous thromboembolism.

CI, 0.12-2.91) for patients with isolated distal DVT. The cumulative incidences of recurrent VTE were $9.52(95 \%$ CI, 7.30-11.74) and 3.86 (95\% CI, 2.61-4.97) per hundred patient-year in provoked and unprovoked VTE, respectively. Of the 39 elderly patients with recurrent VTE, 23 (59\%) were PE with or without DVT, 13 (33\%) were proximal DVT with or without distal DVT and three (8\%) patients experienced isolated distal DVT. Age was categorized into five groups of five-years range (65-69, $70-74,75-79,80-84$, and $\geq 85$ years). The assumption of proportional hazard was not violated (test of equality of survival distributions for the different levels of age category) as this test shows that the hazards for recurrence did not differ by age. Thus, age was not a competing risk factor (advancing age was not associated with increased rates of recurrent VTE). Malignancy-related VTE $(p<0.001)$, immobilisation $(\mathrm{p}=0.043)$, obesity $(\mathrm{p}=0.046)$, and surgery $(p<0.001)$ were significantly higher in the recurrent VTE compared to the group with no recurrent VTE, Table 1.

The initial anticoagulant, duration of therapy, and current medications used are presented in Table 2 . The vast majority of recurrent VTE (36 of 39; 92\%) were diagnosed after anticoagulation treatment had been completed. Of the patients diagnosed with recurrent VTE during anticoagulation therapy (three of 39) one was on warfarin and two on NOAC therapy. The two patients who developed recurrent VTE during NOACs therapy were shifted to warfarin. International normalised ratios (INR) monitoring was followed for all available results to evaluate the magnitude of anticoagulation agent. Of the 208 patients who received vitamin $\mathrm{K}$ antagonist (warfarin), 183 patients (88\%) with regular INR monitoring were available and followed-up (Table 3). The mean percentage of time range in therapeutic $(2-3)$, supratherapeutic $(>3)$, and subtherapeutic $(<2)$ INRs were similar in patients with and without recurrent VTE $(77 \pm 13$ vs $75 \pm 16, p=0.903 ; 23 \pm 15$ vs $19 \pm 13$, $p=0.525 ; 8 \pm 7$ vs $6 \pm 5, p=0.605$, respectively). Patients who developed recurrent VTE during warfarin therapy had subtherapeutic INR. The duration of anticoagulation therapy of patients with and without recurrent VTE were similar ( $p=0.801)$. The majority of patients with no recurrent VTE were using metformin significantly more than those patients with recurrent VTE ( $76 \%$ vs $31 \%, p<0.001$ ). Similarly, antiplatelet use was more frequent in patients with no recurrent VTE than those with recurrent VTE, but was not statistically significant $(57 \%$ vs $41 \%, p=0.061)$, Table 2 .

In multivariate cox regression, malignancy (hazard ratio [HR], 2.87, 95\% $\mathrm{CI}, 1.32-6.24, \mathrm{p}=0.008$ ) and surgery (HR, 2.78, 95\% CI, 1.36-5.67, $\mathrm{p}=0.005$ ) were identified as independent risk factors for recurrent VTE. Metformin had a significant protection effect against recurrent VTE (HR, $0.16,95 \%$ CI, 0.08-0.33, $<<0.001$ ), Table 4.

Overall, seven patients experienced bleeding while receiving anticoagulation (2.5\%); two patients in the recurrent VTE group and five patients in the group with no recurrent VTE (Table 1). All had minor bleeding with no major bleeding reported. Difference in incidence rates was not statistically significant ( $5 \%$ vs $2.1 \%, p=0.281)$. During follow-up, seven (18\%) patients in the recurrent VTE 


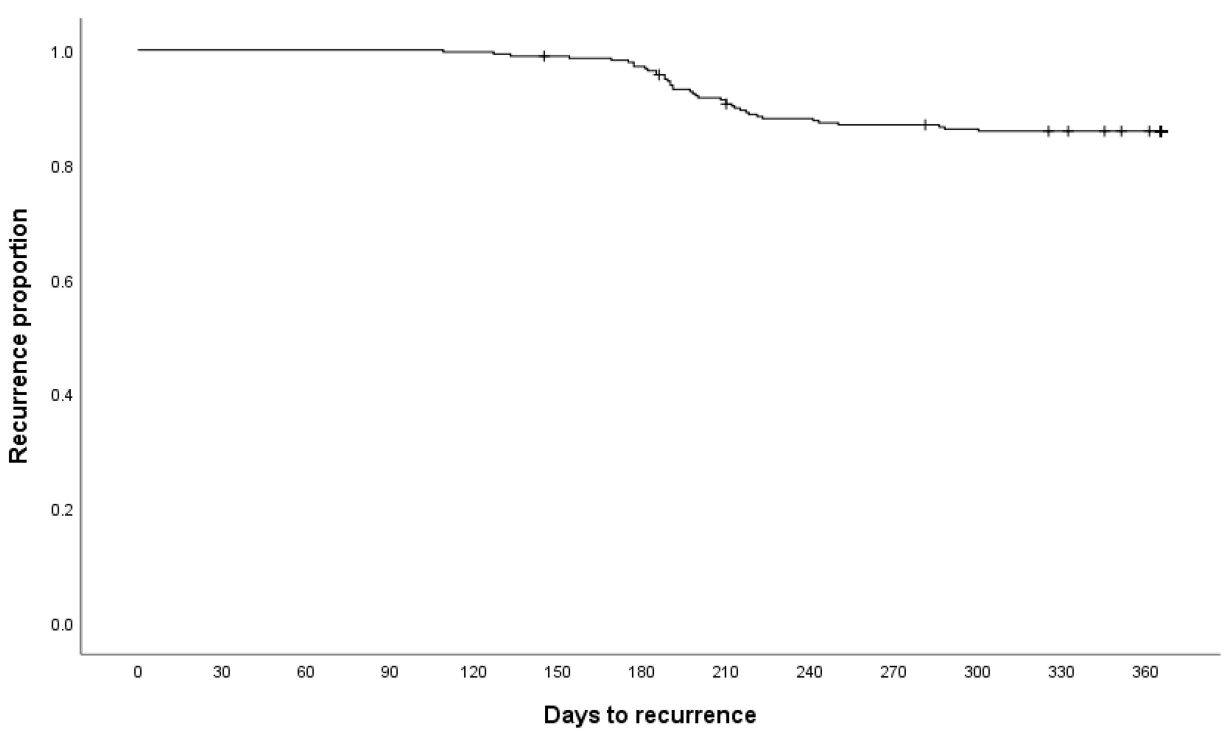

Figure I Kaplan-Meier recurrence probabilities curve.

group, and nine (4\%) in the group with no recurrent VTE died $(\mathrm{p}<0.001$, Table 1$)$.

\section{Discussion}

In this Saudi Arabian prospective follow-up cohort study of the elderly with VTE we found that recurrent VTE was $14 \%$. The cumulative recurrence VTE incidence was 12.75 per hundred patient-year. The incidence rates of first VTE increases with age, ${ }^{2,14,15}$ and was reported that

Table 2 Initial Anticoagulants, Duration and Current Medications

\begin{tabular}{|l|l|l|l|}
\hline Variables & $\begin{array}{l}\text { Recurrent } \\
\text { VTE } \\
(\mathbf{n}=39)\end{array}$ & $\begin{array}{l}\text { No } \\
\text { Recurrent } \\
\text { VTE } \\
(\mathbf{n}=\mathbf{2 3 8})\end{array}$ & P value \\
\hline $\begin{array}{l}\text { Initial anticoagulant agents } \\
\text { Enoxaparin }\end{array}$ & $5(13)$ & $34(14)$ & 0.823 \\
Unfractionated heparin & $1(2)$ & $6(3)$ & \\
Fondaparinux & 0 & $3(1)$ & \\
Warfarin & $30(77)$ & $178(75)$ & \\
NOAC & $3(8)$ & $17(7)$ & \\
\hline Duration of anticoagulant therapy & $5(13)$ & $23(10)$ & \\
3 months & $11(28)$ & $70(29)$ & \\
6 months & $23(59)$ & $145(61)$ & \\
$>6$ months & & & 0.801 \\
\hline Current medications & $16(41)$ & $136(57)$ & 0.858 \\
Antiplatelet & $21(54)$ & $135(57)$ & 0.459 \\
Statin & $18(46)$ & $89(37)$ & $<0.001$ \\
PPI & $12(31)$ & $181(76)$ & \\
Metformin & &
\end{tabular}

Abbreviations: NOAC, non-vitamin K oral anticoagulant; PPI, proton pump inhibitor; VTE, venous thromboembolism. the incidence of VTE increased by 2.5-fold among age group $\geq 80$ years old compared to the age group of 60-69 years old. ${ }^{2}$ However, the risk of recurrent VTE with advanced age is still not fully elucidated, with some studies reporting higher incidence rates, ${ }^{16}$ and others reporting lower incidence rate among patients of advanced age. ${ }^{17}$ The incidence of recurrence rate among our elderly population was higher than in a study of mixed retrospective-prospective cohort of elderly patients $(10 \%),{ }^{18}$ but slightly lower than in prospective and retrospective studies (15\% and $17 \%)$, respectively. ${ }^{19,20}$ However, advanced age ( $\geq 80$ years) in our patients is not associated with increased risk of developing recurrent VTE. Several studies have reported lower risk of recurrent VTE in patients who are aged 80 years or more compared to those aged less than 80 years. $^{2,18,19}$ In a retrospective study of Californian

Table 3 Monitoring the Quality of Vitamin K Antagonist Used by Patients

\begin{tabular}{|c|c|c|c|}
\hline \multirow[t]{2}{*}{ INR Index } & \multicolumn{2}{|c|}{$\begin{array}{l}\text { Mean Percentage of Time in } \\
\text { Different INR Range ( } \pm S D \text { ) }\end{array}$} & \multirow[t]{2}{*}{$P$ value } \\
\hline & $\begin{array}{l}\text { Recurrent } \\
\text { VTE } \\
(n=28)\end{array}$ & $\begin{array}{l}\text { No } \\
\text { Recurrent } \\
\text { VTE } \\
(n=|7|)\end{array}$ & \\
\hline Therapeutic (2.0-3.0) & $77( \pm 13)$ & $75( \pm 16)$ & 0.903 \\
\hline Subtherapeutic $(<2.0)$ & $15( \pm 8)$ & $19( \pm 6)$ & 0.525 \\
\hline Supratherapeutic $(>3.0)$ & $8( \pm 3)$ & $6( \pm 2)$ & 0.605 \\
\hline
\end{tabular}

Abbreviations: INR, international normalized ratio; SD, standard deviation; VTE, venous thromboembolism. 
Table 4 Univariate and Multivariate Cox Regression for Risk Factors Associated with Recurrent VTE

\begin{tabular}{|l|l|l|l|l|}
\hline \multirow{2}{*}{ Variable } & \multicolumn{2}{l|}{ Univariate Analysis } & \multicolumn{2}{l|}{ Multivariate Analysis } \\
\cline { 2 - 5 } & HR (95\% CI) & P-value & HR (95\% CI) & P-value \\
\hline Malignancy & $3.88(1.93-7.80)$ & $<0.001$ & $2.87(1.32-6.24)$ & 0.008 \\
Surgery & $3.49(1.84-6.60)$ & $<0.001$ & $2.78(1.36-5.67)$ & 0.005 \\
Obesity & $2.05(1.04-4.10)$ & 0.039 & $0.92(0.44-1.95)$ & 0.831 \\
Immobilization & $2.24(1.16-4.31)$ & 0.016 & $1.36(0.66-2.84)$ & 0.408 \\
Use of metformin & $0.16(0.08-0.32)$ & $<0.001$ & $0.16(0.08-0.33)$ & $<0.001$ \\
\hline
\end{tabular}

Abbreviations: $\mathrm{Cl}$, confidence interval; $\mathrm{HR}$, hazard ratio.

residents diagnosed with VTE, authors found a $15 \%$ reduction in the incidence rates of recurrent VTE per increasing decade of life. ${ }^{17}$ Similarly, Spencer et al, in their population-based study concluded that advanced age was not associated with increased risk of recurrent VTE. ${ }^{18}$

The VTE recurrence independent risk factors after a first event of VTE in our elderly population study were provoked by malignancy and surgery (HR, 2.87 and 2.78, respectively). Patients with malignancy-associated thrombosis have a higher risk of VTE recurrence of up to $20 \%$, despite receiving anticoagulation. ${ }^{21,22}$ Jara-Palomares et al reported a rate of $8.8 \%(95 \%$ CI: $4.3-15.5 \%)$ developed VTE recurrence within six months in patient with malignancy. ${ }^{23} \mathrm{~A}$ recent study by Nemeth et al of patients undergoing surgery, identified 3,741 patients with one or more prior episodes of treated VTE who were followed for as long as 10 years in a Dutch registry; during follow-up, $580(15.5 \%)$ underwent surgery and $601(16.1 \%)$ developed a recurrent thrombotic event. The 1-month cumulative incidence of recurrent VTE for all surgery types was $2.1 \%$ (95\% CI, $1.2 \%-3.6 \%$ ), which increased to $3.3 \%$ $(95 \% \mathrm{CI}, 2.1 \%-5.1 \%)$ at three months and $4.6 \%(95 \%$ CI, 3.1\%-6.6\%) at six months, compared with an incidence of $0.8 \%(95 \% \mathrm{CI}, 0.6 \%-1.1 \%)$ at three months in patients unexposed to surgery. At six months, risk of recurrent VTE ranged from $2.3 \%$ to $9.3 \%$, depending on surgery type. The authors concluded that surgery was associated with an increased risk of recurrent VTE in patients with a history of VTE; risk remained high for up to six months after the procedure. ${ }^{24}$ The risk of VTE associated with immobilisation is well recognized in surgical and medical patients. ${ }^{25}$ However, immobilisation in this study was not an independent risk factor for recurrent VTE in elderly patients. Immobility was not different in either recurrent or no recurrent VTE groups, most probably due to similarity either in their presenting illness or comorbid conditions. The results of this study show that recurrent VTE is more likely to occur in patients with malignancy and provoked by surgery. Hence, it is important that clinicians are aware of different risk factors that can enhance decision-making discussions with patients about the risks and benefits of the different durations of anticoagulation.

Elderly patients who did not developed VTE recurrence were much more predominantly anticoagulated with antiplatelets (aspirin and clopidogrel) compared with their counterparts with recurrent VTE (73\% vs $41 \%)$. Antiplatelets have some protective effect but did not reach statistical significance $(\mathrm{p}=0.065)$. Similarly, more elderly patients who did not developed VTE recurrence were taking metformin compared to those who developed VTE recurrence $(65 \%$ vs $31 \%)$. Lu et al, in their study, suggested that metformin use had significant protective effects against the development of VTE in patients with diabetes, independent of age, sex, and comorbidities. ${ }^{26}$ Metformin was shown to be effective in preventing both VTE and arterial thrombosis without a significant risk of bleeding. ${ }^{27}$ It acts by inhibiting platelet activation via reducing extracellular mitochondrial DNA (mtDNA) release. Other than decreasing platelet activation, metformin exerts its protective effects by reducing some markers of endothelial activation, von Willebrand factor, vWF, and the adhesion molecule sVCAM-1, ${ }^{27,28}$ and it decreases inflammation and levels of plasminogen activator inhibitor 1 (PAI-1). ${ }^{29-31}$ All these factors put together can account for the anti-thrombotic action of metformin.

The one-year mortality rate of patients who experienced a VTE recurrence (18\%) is a 6.2-fold higher risk of death compared to patients without VTE recurrence (4\%). These findings are consistent with Lauber et $\mathrm{al}^{20}$ who found a similar mortality rate $(20 \%)$ in their study of elderly patients. Moreover, the mortality rate of $18 \%$ observed in our elderly population study is lower than the rate of $26 \%$ in the multicentre Swiss cohort study. ${ }^{32}$ 
This study has several potential limitations. First, the cohort used to perform the analysis was limited to elderly ( $\geq 65$ years old), which may preclude age as a risk factor for recurrent VTE, and we were unable to compare our elderly population data with younger patients. Second, patients with dementia or terminal illness were excluded, which may have an effect on overall mortality. Third, initial treatment in the vast majority (75\%) was prescribed warfarin; therefore our findings may not be generalisable to population prescribed NOACs. Fourth, sample size and the number of events in the present study were relatively small, and may limit generalisability of the findings.

\section{Conclusion}

The findings of this study show that elderly patients with initial VTE have a recurrent rate $(14 \%)$ with a cumulative incidence rate of 12.75 per hundred patient-year. Malignancy and surgery were the most important clinical risk factors to impact significantly the development of recurrent VTE in our elderly population. Metformin may have a protective effect against recurrent VTE in the elderly population, and a larger study is needed to validate our findings.

\section{Ethical Approval}

The study was approved by the Institutional Review Board (IRB) committee at King Abdullah International Medical Research Centre (KAIMRC), Health Affairs, Ministry of National Guard (IRB at KAIMRC is the only committee approves research studies at Health Affairs, Ministry of National Guard). KAIMRC and King Abdulaziz Medical Centre report to the Health Affairs, Ministry of National Guard. All procedures performed in the study involving human participants were in accordance with the ethical standards of the institutional and/or national research committee and with the 1964 Helsinki Declaration and its later amendments, or comparable ethical standards.

\section{Informed Consent}

Informed consent was obtained from all individual participants included in the study.

\section{Acknowledgments}

We gratefully acknowledge Johana Agosto, Vanessa Gallego, and Brian Bolivia for excellent data collection and entry, and Motasim Badri (Professor of Biostatistics) for providing support in the statistical analysis.

\section{Funding}

There is no funding to report.

\section{Disclosure}

The author declares that he has no conflict of interest.

\section{References}

1. Kahn SR, Lim W, Dunn AS. Prevention of VTE in nonsurgical patients: antithrombotic therapy and prevention of thrombosis: American College of chest physicians evidence-based clinical practice guidelines. Chest. 2012;141(suppl2):e195S-e226S. doi:10.1378/ chest.11-2296

2. Anderson FA, Wheeler HB, Goldberg RJ, et al. A population-based perspective of the hospital incidence and case-fatality rates of deep vein thrombosis and pulmonary embolism: the Worcester DVT study. Arch Intern Med. 1991;151:933-938. doi:10.1001/archinte.1991. 00400050081016

3. Stein PD, Hull RD, Kayali F, Ghali WA, Alshab AK, Olson RE. Venous thromboembolism according to age: the impact of an aging population. Arch Intern Med. 2004;164:2260-2265.

4. Engbers MJ, Hylckama AV, Rosendaal FR. Venous thrombosis in the elderly: incidence, risk factors and risk groups. $J$ Thromb Haemost. 2010;8:2105-2112. doi:10.1111/j.1538-7836.2010.03986.x

5. Bauersachs RM. Use of anticoagulants in elderly patients. Thromb Res. 2012;129:107-115. doi:10.1016/j.thromres.2011.09.013

6. Johnson SA, G P E, Rondina MT. Pathogenesis, diagnosis, and treatment of venous thromboembolism in older adults. $J$ Am Geriatr Soc. 2016;64:1869-1878. doi:10.1111/jgs. 14279

7. Hansson PO, Sorbo J, Eriksson H. Recurrent venous thromboembolism after deep vein thrombosis: incidence and risk factors. Arch Intern Med. 2000;160:769-774. doi:10.1001/archinte.160.6.769

8. Schulman S, Lindmarker P, Holmstrom M, et al. Postthrombotic syndrome, recurrence, and death 10 years after the first episode of venous thromboembolism treated with warfarin for 6 weeks or 6 months. J Thromb Haemost. 2006;4:734-742.

9. Baglin T, Luddington R, Brown K, Baglin C. Incidence of recurrent venous thromboembolism in relation to clinical and thrombophilic risk factors: prospective cohort study. Lancet. 2003;362:523-526. doi:10.1016/S0140-6736(03)14111-6

10. Eichinger S, Heinze G, Jandeck LM, et al. Risk assessment of recurrence in patients with unprovoked deep vein thrombosis or pulmonary embolism: the Vienna prediction model. Circulation. 2010;121:1630-1636. doi:10.1161/CIRCULATIONAHA.109.925214

11. Kyrle PA, Minar E, Bialonczyk C, et al. The risk of recurrent venous thromboembolism in men and women. $N$ Engl $J$ Med. 2004;350:2558-2563. doi:10.1056/NEJMoa032959

12. Tosetto A, Iorio A, Marcucci M, et al. Predicting disease recurrence in patients with previous unprovoked venous thromboembolism: a proposed prediction score (DASH). J Thromb Haemost. 2012;10:1019-1025.

13. Schulman S, Kearon C. Subcommittee on control of anticoagulation of the scientific and standardization committee of the international society on thrombosis and haemostasis. Definition of major bleeding in clinical investigations of antihemostatic medicinal products in non-surgical patients. J Thromb Haemost. 2005;3(4):692-694.

14. Spencer FA, Emery C, Lessard D, et al. The Worcester venous thromboembolism study: a population-based study of the clinical epidemiology of venous thromboembolism. J Gen Intern Med. 2006;21(7):722-727. doi:10.1111/j.1525-1497.2006.00458.x

15. Heit JA, Silverstein MD, Mohr DN, et al. The epidemiology of venous thromboembolism in the community. Thromb Haemost. 2001;86(1):452-463. doi:10.1055/s-0037-1616243 
16. Heit JA, Mohr DN, Silverstein MD, Petterson TM, O'Fallon WM, Melton LJ. Predictors of recurrence after deep vein thrombosis and pulmonary embolism: a population-based cohort study. Arch Intern Med. 2000;160(6):761-768. doi:10.10 01/archinte.160.6.761

17. White RH, Zhou H, Romano PS. Length of hospital stay for treatment of deep venous thrombosis and the incidence of recurrent thromboembolism. Arch Intern Med. 1998;158(9):1005-1010. doi:10.1001/archinte.158.9.1005

18. Spencer FA, Gurwitz JH, Schulman S, et al. Venous thromboembolism in older adults: a community-based study. Am J Med. 2014;127 (e533):530-537. doi:10.1016/j.amjmed.2014.02.011

19. Spencer FA, Gore JM, Lessard D, et al. Venous thromboembolism in the elderly. A community-based perspective. Thromb Haemost. 2008;100:780-788. doi:10.1160/TH08-04-0255

20. Lauber S, Limacher A, Tritschler T, et al. Predictors and outcomes of recurrent venous thromboembolism in elderly patients. $\mathrm{Am}$ $J$ Med. 2018;131(6):703.e7-703.e16. doi:10.1016/j.amjmed.2017. 12.015

21. Piran S, Schulman S. Management of recurrent venous thromboembolism in patients with cancer: a review. Thromb Res. 2018;164 (Suppl 1):S172-S177. doi:10.1016/j.thromres.2017.12.019

22. Kearon C, Akl EA, Ornelas J, et al. Antithrombotic therapy for VTE disease: CHEST guideline and expert panel report. Chest. 2016;149 (2):315-352. doi:10.1016/j.chest.2015.11.026

23. Jara-Palomares L, Solier-Lopez A, Elias-Hernandez T, et al. D-dimer and high-sensitivity C-reactive protein levels to predict venous thromboembolism recurrence after discontinuation of anticoagulation for cancer-associated thrombosis. Br J Cancer. 2018;119(8):915-921. doi:10.1038/s41416-018-0269-5

24. Nemeth B, Lijfering WM, Nelissen RGHH, et al. Risk and risk factors associated with recurrent venous thromboembolism following surgery in patients with history of venous thromboembolism. JAMA Netw Open. 2019;2(5):e193690. doi:10.1001/jamanetworkopen.20 19.3690
25. Heit JA, Silverstein MD, Mohr DN, Petterson TM, O'Fallon WM, Melton LJ. Risk factors for deep vein thrombosis and pulmonary embolism: a population-based case-control study. Arch Intern Med. 2000;160(6):809-815. doi:10.1001/archinte.160.6.809

26. Lu DY, Huang CC, Huang PH, et al. Metformin use in patients with type 2 diabetes mellitus is associated with reduced risk of deep vein thrombosis: a non-randomized, pair-matched cohort study. $B M C$ Cardiovasc Disord. 2014;14:187. doi:10.1186/1471-2261-14-187

27. Xin G, Wei Z, Ji C, et al. Metformin uniquely prevents thrombosis by inhibiting platelet activation and mtDNA release. Sci Rep. 2016;6:36222.

28. de Jager J, Kooy A, Schalkwijk C, et al. Long-term effects of metformin on endothelial function in type 2 diabetes: a randomized controlled trial. J Intern Med. 2014;275:59-70. doi:10.1111/ joim. 12128

29. Charles MA, Morange P, Eschwege E, Andre P, Vague P, Juhan-Vague I. Effect of weight change and metformin on fibrinolysis and the von Willebrand factor in obese nondiabetic subjects: the BIGPRO1 study. Biguanides and the prevention of the risk of obesity. Diabetes Care. 1998;21:1967-1972. doi:10.2337/diacare.21.11.1967

30. Derosa G, Gaddi AV, Piccinni MN. Antithrombotic effects of rosiglitazone-metformin versus glimepiride-metformin combination therapy in patients with type 2 diabetes mellitus and metabolic syndrome. Pharmacotherapy. 2005;25(5):637-645. doi:10.1592/ phco.25.5.637.63587

31. Sobel BE, Hardison RM, Genuth S. Profibrinolytic, antithrombotic, and antiinflammatory effects of an insulin-sensitizing strategy in patients in the bypass angioplasty revascularization investigation diabetes (BARI 2D) trial. Circulation. 2011;124:695-703. doi:10.1161/CIRCULATIONAHA.110.014860

32. Faller N, Limacher A, Méan M, et al. Predictors and causes of long-term mortality in elderly patients with acute venous thromboembolism: a prospective cohort study. Am J Med. 2017;130(2):198-206. doi:10.1016/j.amjmed.2016.09.008
Vascular Health and Risk Management

\section{Publish your work in this journal}

Vascular Health and Risk Management is an international, peerreviewed journal of therapeutics and risk management, focusing on concise rapid reporting of clinical studies on the processes involved in the maintenance of vascular health; the monitoring, prevention and treatment of vascular disease and its sequelae; and the involvemen of metabolic disorders, particularly diabetes. This journal is indexed on PubMed Central and MedLine. The manuscript management system is completely online and includes a very quick and fair peerreview system, which is all easy to use. Visit http://www.dovepress. com/testimonials.php to read real quotes from published authors. 